\title{
Cytological Study of Pleural Effusions and its Utility in Clinical Approach
}

\author{
Pravin Gojiya*, Alpeshpuri Goswami and Shaila Shah
}

Department of Pathology, Government Medical College \& Sir Takhtasinhji Hospital, Bhavnagar, India

\section{ABSTRACT}

Background: Cytological analyses of pleural effusions play an important role in the diagnosis of various lesions. Most importantly it gives a significant contribution in diagnosis and management.

Methods: A retrospective study for one year duration from January 2015 to December 2015 was conducted in the Cytology section, Department of Pathology, Govt. Medical College \& Sir- T hospital Bhavnagar. Pleural fluids which were come for cytological analysis from all the departments are included in the study, then physical examination and microscopy done by staining with Gimsa, Papanicolaou stain and Hematoxylin and Eosin Stains for evaluation.

Result: 295 cases of pleural effusion evaluated in which $29 \%$ are female and $71 \%$ are male and $12 \%$ of effusions are Bilateral, $32 \%$ are left sided, 57\% right sided pleural effusion, $95 \%$ non Malignant lesions and only in 5\% of cases were reported as malignant and further non-malignant lesions comprises of pleural effusions with reactive mesothelial cells $11 \%$ while remaining fluids $40 \%$ are of lymphocytic predominant effusions and $13 \%$ are of acute inflammatory conditions with abundant polymorphs, remaining $36 \%$ of pleural effusions were scant-cellular and have no suggestive role by cytological evaluation.

Conclusion: This study showed that meticulous evaluation of the pleural fluids for their cytological properties will help the clinicians in clinching the diagnosis as well as early approach in management of these cases.

Keywords: Pleural Fluid Cytology, Benign Effusion, Reactive Mesothelial Cells, Malignant Effusions

\section{Introduction}

Pleural fluid cytology is a simple and a minimally invasive technique as the preliminary step for the diagnostic evaluation of pleural effusions, assisting the clinician in establishing the differential diagnosis. It may lead to final diagnosis and provide useful information for treatment. The diagnostic yield of the cytological analysis may be attributable to the cell population present in the sediment that is representative of a much larger surface area than the pleural biopsy ${ }^{[1,2]}$. It is very useful in the very first initial work up for the management of the case of pleural effusion. It may also provide crucial clues for the identification of both non-malignant pleural and transudative effusions. Sensitivity will be higher if the clinical, radiological and laboratory results are collaborated. This study was carried out to determine the diagnostic utility of pleural fluid cytology.

\section{Materials and Methods}

The study was conducted in the Department of PathologyCytology section of Govt. Medical College and Sir T Hospital, Bhavnagar (Gujarat). It's a retrospective study of cytology impression of pleural fluids for one year duration from January 2015 to December 2015. Pleural fluids which received for cytological analysis from the various departments of our institute with the proper complete history and clinical brief are included in the study. These fluid samples were examined for physical properties like the volume, color, appearance, turbidity, presence of clot coagulum. Fluids samples are then centrifuged at $2000 \mathrm{rpm}$ for five minutes. Smears made from the sediment part of centrifuged fluid and stained with Giemsa, Hematoxylineosin and Papanicolaou stains. Smears were examined for the differential cell count and reported descriptively, final impression given as malignant or non-malignant pleural effusion. Malignant pleural effusions were further classified according to its morphometrics. Study results then analyzed with the help of tables and charts and discussed for its incidence rate.

\section{Result}

In our study during the year 2015 total 295 cases of pleural fluids were examined for cytology. Out of 295 pleural fluids specimens 84 samples were of females and remaining 211 were of males, so here male is to female ratio of pleural fluid specimens was 2.5:1CHART-1. The pulmonary department had sent pleural fluid examination mostly among the all other departments. Pleural effusions 
occurrence was more common in the age group 60 to 70 years in both males and females sexes although it occurs in all age group from 9 years to 90 years of age $\boldsymbol{C H} \boldsymbol{A R T}$-2. Out of 295 cases 164 cases were of right sided, 96 cases were left sided pleural effusions and remaining 35 cases were of bilateral pleural effusion. About $95 \%$ of pleural fluids were non-malignant effusions given in pleural fluid cytology, here out of 295 cases 280 , comprises $36(13 \%$ ) of acute

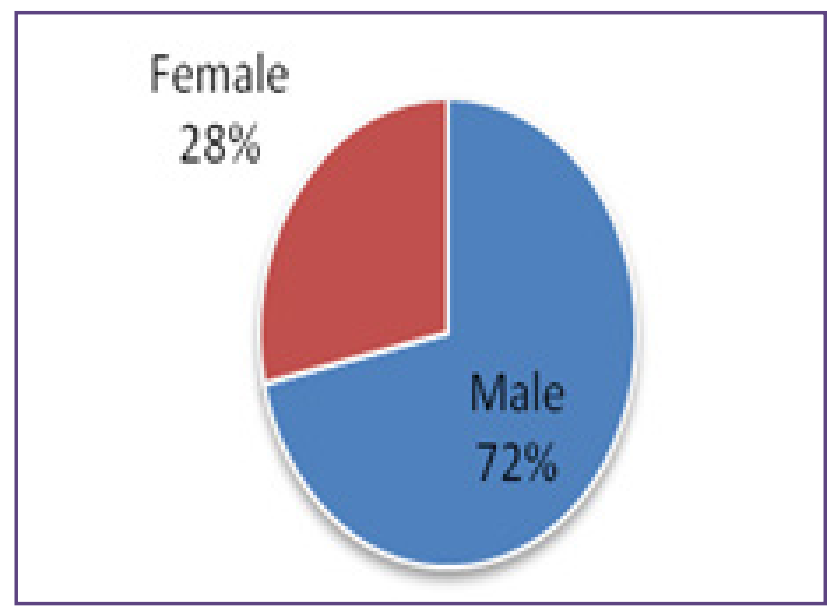

Chart 1: Sex Distribution of Pleural effusion cases inflammations with neutrophilic preponderance(fig.1), $112(40 \%)$ with lymphocytic(fig.2), 30(11\%) effusions have reactive mesothelial cells(fig.4) and remaining 102(36\%) cases have no further suggestive outcome in pleural fluid cytology. Malignant pleural effusions were $15(5 \%)$ out of which, 6(40\%) metastatic adenocarcinoma (fig.3) and remaining pleural effusions have non-specific malignant cells effusions $T A B L E-1$.

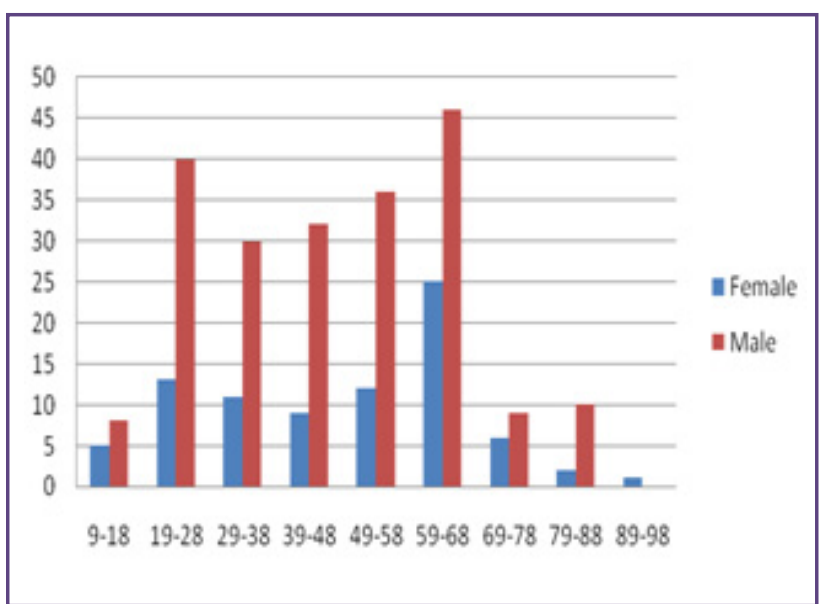

Chart 2: Age distribution in both sexes Vs frequencies for pleural effusions

Table 3: Diagnostic outcome of pleural fluid cytology examinations.

\begin{tabular}{|l|l|c|}
\hline & Diagnostic impression & Number of cases \\
\hline \multirow{3}{*}{ Malignant pleural effusion (5\%) } & Malignant metastatic adenocarcinoma (20\%) & 3 \\
\cline { 2 - 3 } & Malignant effusion (40\%) & 6 \\
\cline { 2 - 3 } & Lymphoma (40\%) & 6 \\
\hline \multirow{4}{*}{ Non-Malignant pleural effusion (95\%) } & Acute inflammatory effusion(13\%) & 36 \\
\cline { 2 - 3 } & Lymphocytic effusion (40\%) & 112 \\
\cline { 2 - 3 } & Reactive mesothelial effusion (11\%) & 30 \\
\cline { 2 - 3 } & Non specific effusion(36\%) & 102 \\
\hline
\end{tabular}

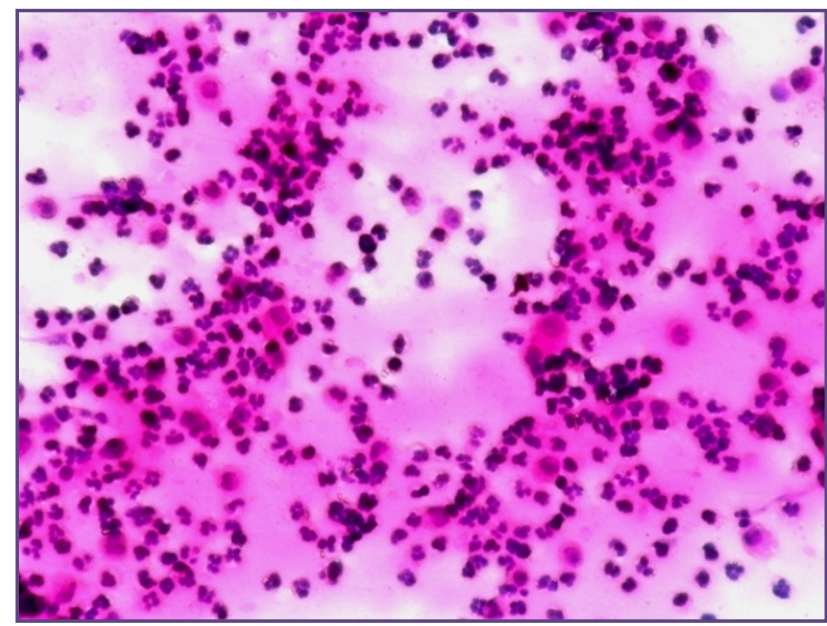

Fig. 1: Centrifuged smear of pleural fluid shows Plenty of Polymorphs-Acute inflammation [H\&E, 40X].

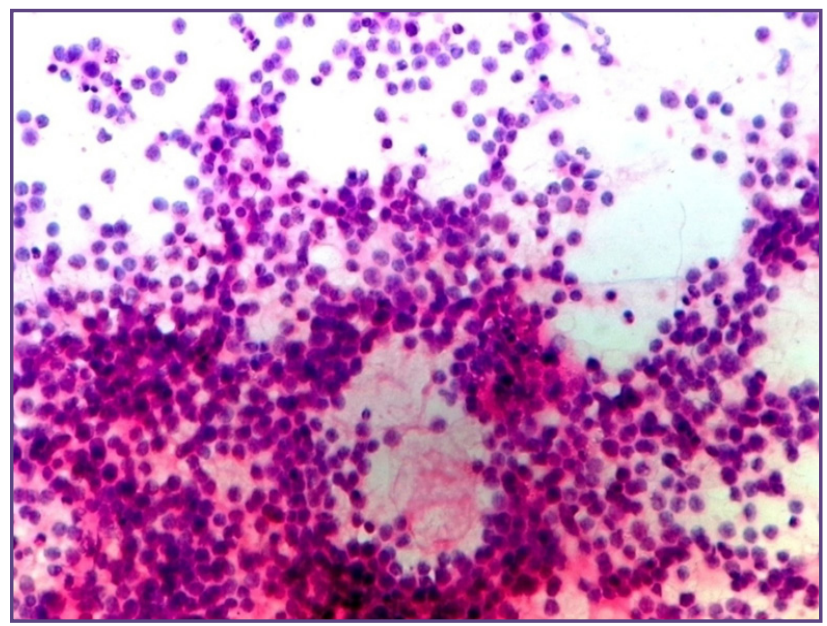

Fig. 2: Centrifuged smear of pleural fluid shows plenty of lymphocytes - Chronicinflammation [H\&E, 40X]. 


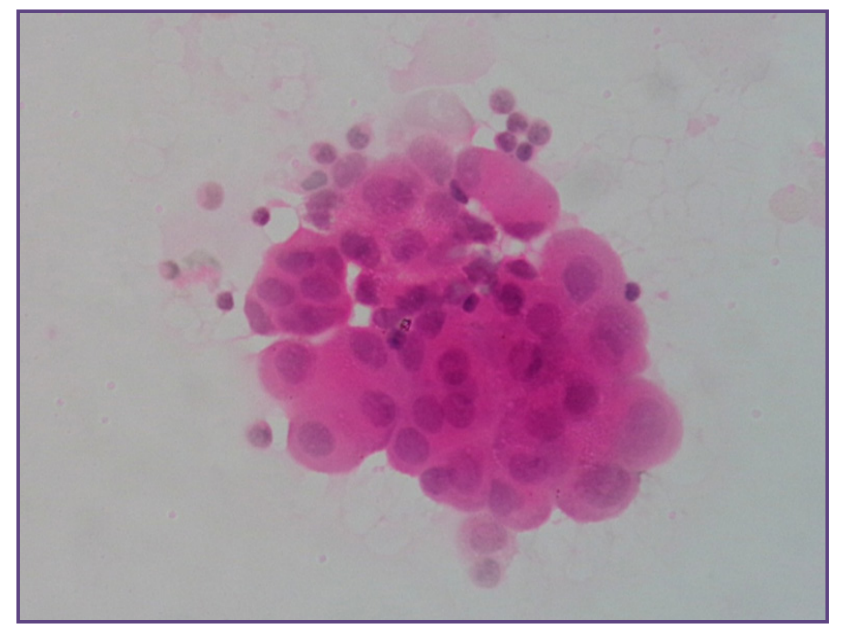

Fig. 3: Malignant cells in Pleural fluid (Adenocarcinoma). Cells arranged in 3D ball pattern, shows pleomorphism \& high $\mathrm{N}$ :C ratio[H\&E, 40X].

\section{Discussion}

Pleural effusions are caused by pulmonary or nonpulmonary diseases. Although the etiologic spectrum is wide, most effusions occur due to malignancy, heart failure, tuberculosis or bacterial infection. ${ }^{[1,7,8,9]}$ Because the effusions develop as a manifestation of an underlying disease, it is difficult to determine the precise incidence. Cytological examination is often the initial diagnostic step for its etiologic identification. It represents the whole pleural surface due to the existence of exfoliated cells in the fluid. Even if the initial examination is negative, the cellular profile of the fluid leads the clinician in the correct diagnostic pathway, as the results of our study suggests. Consequently, this minimally invasive method appears to be the best preliminary step for the assessment of pleural effusions. In patients with a malignant pleural effusion, cytological examination is a fast, efficient and minimally invasive procedure to establish the diagnosis.

Almost all adenocarcinomas were diagnosed with cytology, but the yield was less with squamous cell carcinomas, Hodgkin's disease and sarcomas ${ }^{[1]}$. This may be due to the fact that the adenocarcinoma cells are more easily identified cytological and large tissue biopsies are usually needed for the diagnosis of lymphoma and sarcomas. The effusion may develop secondary to other factors such as infection, pulmonary emboli or lymphatic blockade. Even after thoracoscopy, $10 \%$ of the pleural effusions may remain undiagnosed ${ }^{[12,13]}$. A tuberculous pleural effusion is frequently a diagnostic challenge for the pulmonary clinician. It is impossible to differentiate a tuberculous effusion from a malignant pleural effusion on clinical grounds alone and usually more invasive diagnostic interventions are needed. As the results of our

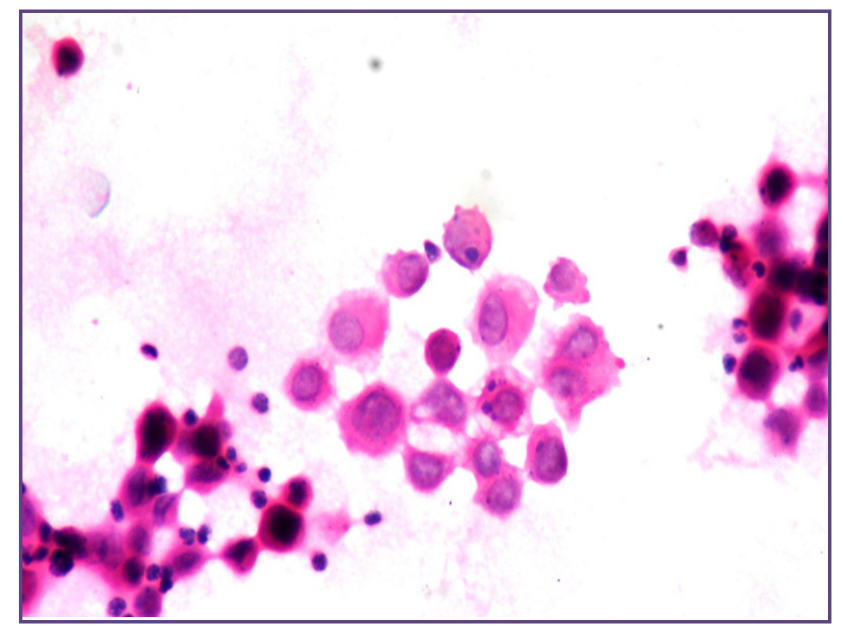

Fig. 4: Centrifuged smears of pleural fluid shows Reactive mesothelial cells with high $\mathrm{N}: \mathrm{C}$ ratio, vacuolated Eosinophilic cytoplasm [H\&E, 40x]

study suggests, the absence or the scarcity of mesothelial cells along with the presence of more than $50 \%$ small lymphocytes should be regarded as a strong evidence for tuberculosis ${ }^{[1]}$. The absence of mesothelial cells is attributed to the deposition of fibrin on the pleural surface, either sealing off the mesothelial cells, destroying them or both ${ }^{[14]}$. Although the mechanism of eosinophil accumulation in the pleural space is unknown, eosinophils play an important role in idiopathic, allergic diseases, or drug reactions. The presence of air in the pleural space may also cause eosinophilia. It is well known that tuberculous pleural effusions rarely contain more than $10 \%$ eosinophils ${ }^{[15,16,17,18]}$. The presence of numerous mesothelial cells or eosinophils was useful to exclude tuberculosis in the differential diagnosis of exudative pleural effusions. In the present study, pleural fluid analysis was not diagnostic in approximately $30 \%$ of the patients and thereby indicating its limits.

As the results of our study suggests, cytology of the pleural fluid is the most informative and definitive initial diagnostic step in pathologic states involving the pleura. This simple and minimally invasive technique may be considered as the best initial diagnostic tool in the hands of an experienced cytologist without any serious complications of thoracocentesis. Examination of the pleural fluid can narrow the differential diagnosis considerably. Cytology can be the key to direct diagnosis or can indicate the next step leading the clinician in the correct pathway for final diagnosis even when not diagnostic on its own, and thus precluding unnecessary invasive interventions. In most diseases related to pleural effusion, the pleural fluid analysis yields important diagnostic information and in certain cases it provides the final diagnosis ${ }^{[1]}$. 


\section{Conclusion}

In developing countries like ours, where investigations and health facilities are inadequate and cost of treatment is often un-affordable, pleural fluid analysis and cytology should continue to be a first line investigation to screen out the suspiciously malignant pleural effusion cases, as it is a very convenient, cost-effective and safe investigation. Pleural fluid cytology may be helpful in diagnosing primary as well as metastatic pleural malignancies. However, along with the proper clinical history and other supportive radiological, biochemical investigations the diagnostic impression of the suspicious disease can be achieved satisfactorily for further clinical management.

\section{Acknowledgements}

We are very much thankful to pulmonologists of Sir Takhtasinhji hospital for their valuable support.

\section{Reference}

1. Tetikkurt C, Y1lmaz Kara B, Tetikkurt S, Y1lmaz N, Ilknur Yasar Rian Disci. The Value of Cytology in the Diagnosis of Pleural Effusions British Journal of Medicine \& Medical Research 2014;4(11): 2203-11

2. Kushwaha R, Shashikala P, Hiremath S, Basavaraj HG. Cells in pleural fluid and their value in differential diagnosis. J Cytol. 2008;25(4):138-143.

3. BedrossianW. Diagnostic problems in serous effusions. Diagn Cytopathol.1998;19(2): 131-137.

4. Pereira TC, Saad RS, Liu Y, Silverman JF. The diagnosis of malignancy in effusion cytology: A pattern recognition approach. Adv Anat Pathol. 2006;13:174-184.

5. Awasthi A, Gupta N, Srinivasan R, Nijhawan R, Rajwanshi A. Cytopathologic spectrum of unusual malignant effusions at a tertiary care centre in North India. Cytopathol. 2007; 18:28-32.2211

6. Geisinger KR, Stanley MW, Raab SS, Siverman JF, Abati A. Modern Cytopathology. Philadelphia Churchill Livingstone; 2004.

7. Sahn SA. Pleural effusions of extravascular origin. Clin Chest Med. 2006;27(2):285-308.

8. Light RW. The undiagnosed pleural effusion. Clin Chest Med. 2006;27(2):309-319.

9. Heffner JE. Diagnosis and management of malignant pleural effusions. Respirology. 2008;13(1):5-20.

10. Bouros D, Pneumatikos I, Tzouvelekis A. Pleural involvement in systemic autoimmune disorders. Respiration. 2008;75(4):361-71.

11. Dekker A, Bupp PA. Cytology of serous effusions. An investigation into the usefulness of cell blocks versus smears. Am J ClinPathol. 1978;70:855-860.

12. Loddenkemper R, Boutin C. Thoracoscopy diagnostic and therapeutic indications. EurRespir J. 1993;6:1544-1555.

13. Kalomenidis J. New advances in the investigation of pleural diseases. Pneumologie. 2003;16:247-251.

14. Hurwitz S, Leiman G, Shapiro C. Mesothelial cells in pleural fluid TB or not TB. S Afr Med J. 1980;57:937-939.

15. Epstein DM, Kline RM, Albelda SM, Miller WT. Tuberculous pleural effusions. Chest. 1987;91:106-109.

16. Light RW. Establishing the diagnosis of tuberculous pleuritis. Arch Intern Med 1998;158:1967-1968.

17. Sahn SA. The value of pleural fluid analysis. Am J Med Sci. 2008;335(1):7-15.

18. Sakuraba M, Masuda K, Hebisawa A, Sagara Y, Komatsu H. Pleural effusion adenosine deaminase (ADA) level and occult tuberculous pleurisy. Ann Thorac Cardiovasc Surg. Oct 2009;15(5):294-6.

*Corresponding author:

Dr. Pravin Gojiya, Room no. 27, PG Hostel-1, Sir-T hospital campus, kala nala, Bhavnagar-364001,India Phone: +91 0278-2510236

Email: pgojiya@gmail.com

Financial or other Competing Interests: None.

Date of Submission : 10.01.2017

Date of Acceptance : 31.05.2017

Date of Publication : 31.08.2017 